\title{
Elevated serum free light chains predict cardiovascular risk in type 1 diabetes mellitus
}

\author{
LAUREN MARIE QUINN, ${ }^{1}$ SHERIDAN MCWILLIAM, ${ }^{1}$ JOHN P CAMPBELL, ${ }^{2}$ YAN WANG, ${ }^{2}$ \\ DAVID HUGHES, ${ }^{3}$ MARK T DRAYSON, ${ }^{2}$ PARTH NARENDRAN ${ }^{3}$
}

\begin{abstract}
Aims: Increased serum polyclonal combined immunoglobulin free light chain ( $C F L C=F L C K+F L C \lambda$ ) has been shown to predict cardiovascular (CV) events in South Asians with type 2 diabetes mellitus. We examined whether CFLC also predicted $\mathrm{CV}$ risk in unselected patients with type 1 diabetes mellitus. Methods: CFLC was estimated in the serum of 55 adults with type 1 diabetes mellitus. CV risk was measured through two validated risk engines: Q-Risk and PROCAM. Statistical association was tested using the parametic Pearson's or the Spearman's rank correlation coefficient test, Student t-test or Mann-Whitney U-test and Kruskall-Wallis test for parametric or non-parametrically distributed data accordingly.

Results: CFLC was associated with CV risk. This association was significant when estimated through either risk engine (PROCAM $p=0.003$, $Q-R i s k p=0.012$ ). cFLC was associated with diabetes mellitus duration $(p=0.003)$, age $(p=0.006)$ and history of cardiac disease $(p=0.042)$.

Conclusions: These findings indicate that cFLC is a marker of $\mathrm{CV}$ risk in people with type 1 diabetes mellitus. Moreover, it supports emerging data demonstrating $\mathrm{CFLC}$ as a prognostic indicator for mortality.

Br J Diabetes 2016;16:176-178
\end{abstract}

Key words: free light chains, cardiovascular disease, type 1 diabetes mellitus

\section{Introduction}

Immunoglobulins are comprised of two heavy and two light chains, although the latter are synthesised in excess by the plasma cells hence existing as combined free light chains (CFLC, sum of total FLCK and FLC $\lambda$, the two light chain isoforms) in the serum. ${ }^{1}$

The Medical School, University of Birmingham, Birmingham, UK Clinical Immunology Service, The Medical School, University of Birmingham, Birmingham, UK

Clinical and Experimental Medicine, Institute of Biomedical Research, The Medical School, University of Birmingham, Birmingham, UK

Address for correspondence: Dr Parth Narendran

Clinical and Experimental Medicine, Institute of Biomedical Research, The Medical School, University of Birmingham, Birmingham B15 2TT, UK

Tel: +44 (0)1216278904

E-mail: p.narendran@bham.ac.uk

http://dx.doi.org/10.15277/bjd.2016.107
Monoclonal FLC - or Bence Jones proteins - are elevated in multiple myeloma ${ }^{1}$ whereas increased polyclonal cFLC is associated with inflammatory conditions and disease activity, as demonstrated in rheumatoid arthritis and systemic lupus erythemato-

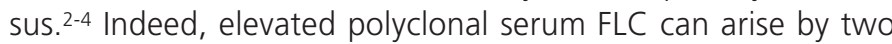
principal mechanisms: renal impairment and/or chronic immune stimulation, as observed in diabetes patients with cardiovascular disease, ${ }^{5}$ which are all prominent complications of diabetes. Furthermore, FLC have been shown to exert independent biological functions rather than conferring simple bystander effects. ${ }^{6}$

Elevated CFLC are associated with increased mortality in the general population 7,8 and are predictive of cardiovascular events in South Asian patients with type 2 diabetes mellitus. ${ }^{9}$ People with type 1 diabetes mellitus differ from those with type 2 diabetes mellitus because they are younger, and their condition results from a chronic autoimmune inflammatory destruction of beta cells. We examined whether CFLC also predicted cardiovascular risk in patients with type 1 diabetes mellitus.

\section{Methods}

We explored CFLC and cardiovascular risk in patients with diabetes from the Chronic Disease Research (CDR) into Diabetes study. ${ }^{10}$ The CDR is based in the West Midlands region of the UK and is designed to explore the natural history of diabetes and its complications. Demographic (age, gender, diabetes and smoking history, medical therapy, past and family history of heart disease, medical history) and clinical (BP, height, weight) data as well as biological samples were obtained from all consenting and participating patients. The Q-Risk and PROCAM scores were calculated to determine each patient's risk of cardiovascular disease. These are validated risk engines for estimating cardiovascular risk and allow inclusion of the cardiovascular risk associated with diabetes.

Serum CFLC were measured on a monoclonal antibody-based multiplex assay, as previously described, ${ }^{11}$ using a Luminex ${ }^{\circledR}$ instrument where the fluorescent signal intensity was inversely correlated with $\mathrm{k}$ or $\lambda$ FLC concentration. ${ }^{6}$ This assay has good sensitivity and reproducibility. ${ }^{11}$

We undertook parametric statistical analyses using Excel 2013. Pearson's correlation coefficient was used to determine correlations between CFLC and continuous variables such as age and duration of diabetes. For categorical variables with two groups (e.g. presence or absence of cardiac disease), an F-test was used to determine variance between the two groups, followed by an equal or unequal variance t-test to determine 
Figure 1. Association of serum CFLC with cardiovascular risk estimated through two risk engines (PROCAM and Q-Risk)

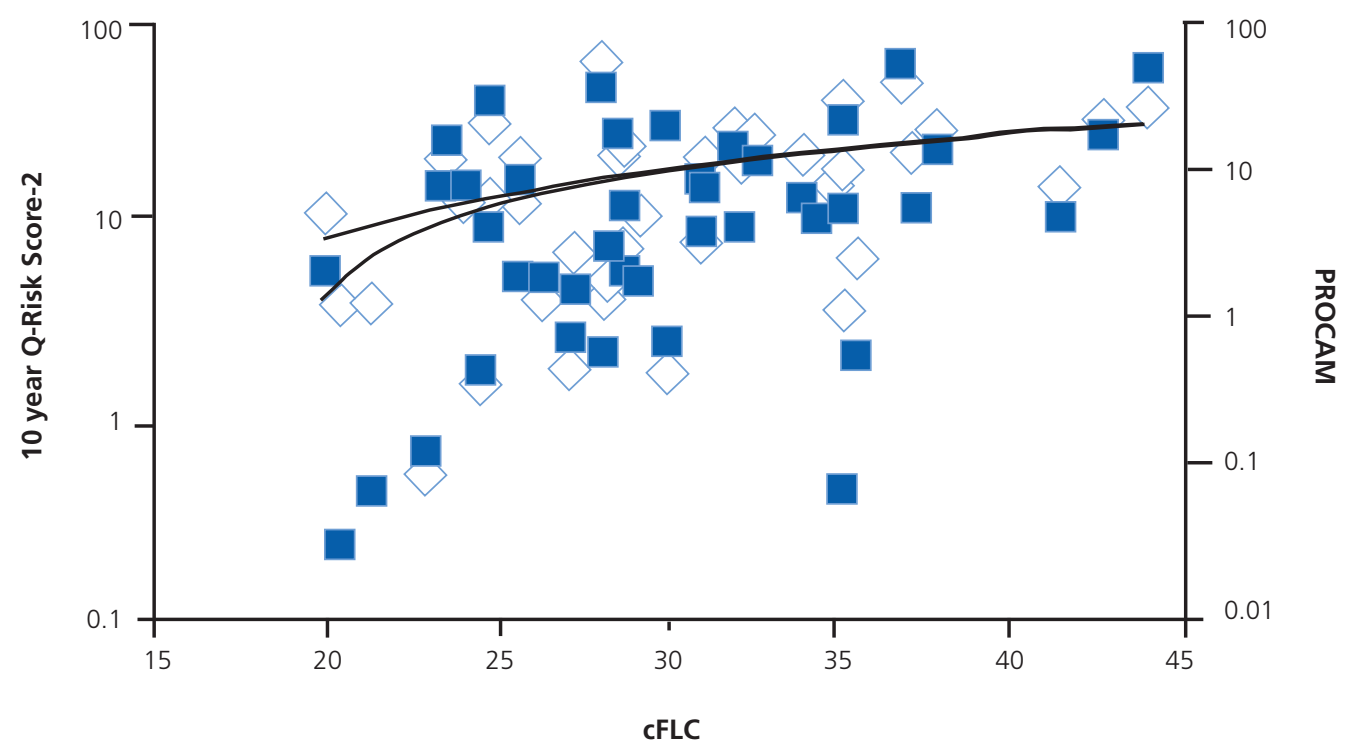

Q-Risk

significant differences in CFLC between the groups. An ANOVA test was performed for categorical variables with more than two groups.

Non-parametric statistical analysis using SPSS V.9 software was then undertaken to confirm the reliability of these findings. The tests used were Spearman's rank correlation coefficient for continuous variables, Mann-Whitney U-test for categorical variables with two groups and the Kruskall-Wallis test for categorical variables with more than two groups.

Age data were available in the CDR database as an age range only. Hence, given the ordinal nature of these age data, a Jonckheere-Terpstra test was performed, which is a rank-based nonparametric statistical test used to determine any significant differences between the continuous variable cFLC and the ordinal age groups.

\section{Results}

cFLC was measured in 55 patients with type 1 diabetes mellitus (28 male, mean age 47). The majority (92\%) were white Caucasians. FLCK and FLC $\lambda$ ratios were not abnormal in any of the patients, thus excluding monoclonal gammopathy as an underlying cause for increased CFLC. All data required for analysis were available for 44 of the 55 patients.

CFLC was associated with duration of diabetes $\left(r^{2}=0.127\right.$ $p=0.003$, assessed as a continuous variable), age $(p=0.006$, assessed using ordinal data) and history of cardiac disease $(p=0.042)$. cFLC was not associated with smoking, statin or BP therapy, family history of ischaemic heart disease, $\mathrm{HbA}_{1 \mathrm{c}}$, ethnicity or body mass index. Unlike type 2 diabetes mellitus, 9 there was no association between cFLC and cholesterol $\left(r^{2}=0.029\right.$, $p=0.750)$ or systolic BP $\left(r^{2}=-0.099 p=0.140\right)$ in type 1 diabetes mellitus.
Serum cFLC was strongly associated with cardiovascular risk, and this association was significant when estimated through either risk engine (PROCAM: $r^{2}=0.393, p=0.003$; Q-Risk: $r^{2}=0.408, p=0.012$; Figure 1).

Furthermore, the significant associations between cFLC and diabetes duration, PROCAM and Q-risk 10-year scores remained after patients with a past medical history of ischaemic heart disease were excluded from the parametric and non-parametric statistical analyses.

There was no association between elevated cFLC and renal disease as measured by urinary albumin creatinine ratio.

\section{Discussion}

We show for the first time in people with type 1 diabetes mellitus that CFLC are associated with the risk of cardiovascular disease. This supports findings from a similar study in a defined ethnic subpopulation with type 2 diabetes mellitus ${ }^{9}$ and expands this to a more generalised UK population. It also supports studies in the general population where CFLC are associated with the risk of all-cause mortality. ${ }^{7,8}$ The potential for reverse causation was addressed through a separate analysis that excluded patients with an established history of ischaemic heart disease.

However, this remains a cross-sectional study and needs to be validated in a larger cohort of patients and in a prospective manner. cFLC may play an important role in refining current risk algorithms of cardiovascular risk in people with type 1 diabetes mellitus. Cardiovascular disease is the commonest cause of death in type 1 diabetes mellitus in the Western world, and current risk engines are based on studies in the general population or in people with type 2 diabetes. Potential biomarkers of cardiovascular risk in patients with type 1 diabetes are therefore worthy of further investigation. 


\section{Key messages}

- Elevated Free Light Chains are a marker of cardiovascular risk in patients with type 1 diabetes

- Increased duration of diabetes, advancing age and cardiovascular disease are associated with elevated Free Light Chains in patients with type one diabetes

- Free Light Chains is a prognostic indicator of morbidity and mortality in type one diabetes and warrants further investigation

CFLCs are manufactured and secreted by B cell lymphoid lineage cells during normal immunoglobulin production, where nearly twice as many light chains are generated in the cytoplasm compared with heavy chains..$^{12}$ It is proposed that this prevents toxicity from free heavy chain accumulation. ${ }^{13}$ The serum cFLC reference range in healthy donors is approximately 3-19 $\mathrm{mg} / \mathrm{L}$ for FLCK and 6-26 mg/L FLC $\lambda .{ }^{14}$ Serum polyclonal CFLC may be elevated above normal by two principal mechanisms: renal impairment and immune stimulation. Unlike whole immunoglobulin that is cleared from the bloodstream by cellular catabolism with a half-life of approximately one week (IgA, $\lg M)$ to three weeks $(\mathrm{IgG})$, cFLCs are cleared by the renal glomeruli and metabolised in the proximal tubules of the nephrons and have a shorter half-life of approximately two hours ( $\mathrm{k}$ ) to four hours $(\lambda)$. Thus, as a consequence of renal impairment, serum CFLC are elevated and show strong positive correlations with creatinine, cystatin- $C$ and disease staging (e.g. chronic kidney disease). ${ }^{2}$ There was no evidence in our analysis to suggest that the elevated CFLC could be explained by renal impairment.

cFLCs may also be secreted to exert independent biological functions. ${ }^{6}$ As such, chronic activation of the immune system, as is seen with cardiovascular disease, gives rise to elevated levels of both whole immunoglobulin and CFLC. Therefore, the chronic inflammation associated with cardiovascular risk may explain the increased CFLC seen in our study.

The association we describe may potentially be causative. CFLCs may possess an atherogenic property that contributes independently to the pathogenesis and acceleration of cardiovascular disease rather than simply acting as a biomarker of disease. Either way, this analysis suggests that understanding the association between elevated CFLC and cardiovascular risk in type 1 diabetes mellitus could refine current risk stratification strategies for cardiovascular disease and/or herald novel therapies to ameliorate the condition.

\section{Conflict of interest None Funding None}

Contributors LMQ and SM contributed equally to the paper. LMQ and SM undertook the statistical analyses, the data interpretation and created the figure.
LMQ and SM wrote the abstract and the statistical component of research design and methods. PN wrote the first draft of the article and performed intensive review of the literature. JPC collected and measured the serum light chain samples and edited the article. YW measured the CFLC. DH managed the CDR. All authors, including MTD, revised critically and approved the final manuscript. PN acts as guarantor, taking responsibility for the contents of the article.

\section{References}

1. van der Heijden M, Kraneveld A, Redegeld F. Free immunoglobulin light chains as target in the treatment of chronic inflammatory diseases. Eur J Pharmaco/ 2006;533:319-26. https://doi.org/10.1016/j.ejphar.2005.12.065

2. Hutchison CA, Harding S, Hewins $P$, et al. Quantitative assessment of serum and urinary polyclonal free light chains in patients with chronic kidney disease. Clin J Am Soc Nephrol 2008;3:1684-90. http://dx.doi.org/10.2215/CJN.02290508

3. Sølling K, Sølling J, Rømer FK. Free light chains of immunoglobulins in serum from patients with rheumatoid arthritis, sarcoidosis, chronic infections and pulmonary cancer. Acta Med Scand 1981;209:473-7. https://doi.org/10.1111/j.0954-6820.1981.tb11632.x

4. Draborg AH, Lydolph MC, Westergaard M, et al. Elevated concentrations of serum immunoglobulin free light chains in systemic lupus erythematosus patients in relation to disease activity, inflammatory status, B cell activity and Epstein-Barr virus antibodies. PLoS One 2015;10:e0138753. http://dx.doi.org/10.1371/journal.pone.0138753

5. Festa A, D'Agostino R, Howard G, Mykkänen L, Tracy RP, Haffner SM. Inflammation and microalbuminuria in nondiabetic and type 2 diabetic subjects: The Insulin Resistance Atherosclerosis Study. Kidney Int 2002;58:1703-10. https://doi.org/10.1046/j.1523-1755.2000.00331.x

6. Hutchinson AT, Jones DR, Raison RL. The ability to interact with cell membranes suggests possible biological roles for free light chain. Immunol Lett 2012;142:75-7. http:dx.doi.org/10.1016/j.imlet.2011.10.013

7. Dispenzieri A, Katzmann JA, Kyle RA, et al. Use of nonclonal serum immunoglobulin free light chains to predict overall survival in the general population. Mayo Clin Proc 2012;87:517-23. http://dx.doi.org/10.1016/j.mayocp.2012.03.009

8. Anandram S, Assi LK, Lovatt T, et al. Elevated, combined serum free light chain levels and increased mortality: a 5-year follow-up, UK study. J Clin Pathol 2012;65:1036-42. http://dx.doi.org/10.1136/jclinpath-2012-200910

9. Bellary S, Faint JM, Assi LK, et al. Elevated serum free light chains predict cardiovascular events in type 2 diabetes. Diabetes Care 2014;37:202830. http:dx.doi.org/10.2337/dc13-2227

10. University of Birmingham. Chronic Disease Research into Diabetes Study. 2015. Available from: http://www.birmingham.ac.uk/research/ activity/mds/trials/pccrtu/trials/cdr/index.aspx (accessed 21 Jan 2015).

11. Campbell JP, Cobbold M, Wang Y, et al. Development of a highly-sensitive multi-plex assay using monoclonal antibodies for the simultaneous measurement of kappa and lambda immunoglobulin free light chains in serum and urine. J Immunol Methods 2013;391:1-13. http://dx.doi.org/10.1016/j.jim.2013.01.014

12. Hopper JE, Papagiannes E. Evidence by radioimmunoassay that mitogen-activated human blood mononuclear cells secrete significant amounts of light chain Ig unassociated with heavy chain. Cell Immunol 1986;101:122-31. https://doi.org/10.1016/0008-8749(86)90191-7

13. Corcos D, Osborn MJ, Matheson LS, et al. Immunoglobulin aggregation leading to Russell body formation is prevented by the antibody light chain. Blood 2010;115:282-8. http://dx.doi.org/10.1182/blood-2009-07-234864

14. Katzmann JA, Clark RJ, Abraham RS, et al. Serum reference intervals and diagnostic ranges for free kappa and free lambda immunoglobulin light chains: relative sensitivity for detection of monoclonal light chains. Clin Chem 2002:48:1437-44 